\title{
Role of Filler Content and Morphology in LLZO/PEO Membranes
}

\begin{abstract}
Mir Mehraj Ud Din ${ }^{1,2,3 *}$, M. Häusler ${ }^{1}$, S. M. Fischer ${ }^{3,4}$, K. Ratzenböck $^{3,4}$, F. F. Chamasemani ${ }^{5}$, I. Hanghofer ${ }^{6}$, V. Henninge ${ }^{6}$, R. Brunner ${ }^{5}$, C. Slugovc ${ }^{3,4}$ and D. Rettenwander ${ }^{1,2,3 *}$

${ }^{1}$ Department of Materials Science and Engineering, NTNU Norwegian University of Science and Technology, Trondheim, Norway, ${ }^{2}$ International Christian Doppler Laboratory for Solid-State Batteries, NTNU Norwegian University of Science and Technology, Trondheim, Norway, ${ }^{3}$ Institute of Chemistry and Technology of Materials (NAWI Graz), Graz University of Technology, Graz, Austria, ${ }^{4}$ Christian Doppler Laboratory for Organocatalysis in Polymerization, Graz University of Technology, Graz, Austria,

${ }^{5}$ Materials Center Leoben, Forschung GmbH, Leoben, Austria, ${ }^{6}$ AVL List GmbH, Graz, Austria
\end{abstract}

Polymer electrolytes containing Li-ion conducting fillers are among the extensively investigated materials for the development of solid-state Li metal batteries. The practical realization of these electrolytes is, however, impeded by their low Li-ion conductivity, which is related to the filler and the interplay between the filler and the polymer. Therefore, we performed an in-depth analysis on the influence of the filler

OPEN ACCESS

Edited by:

Asma Sharafi,

Ford Motor Company, United States

Reviewed by:

Wyatt Tenhaeff,

University of Rochester, United States

Eric Kazyak,

University of Michigan, United States

*Correspondence:

Mir Mehraj Ud Din

mir.m.u.din@ntnu.no

D. Rettenwander

daniel.rettenwander.ntnu.no

Specialty section:

This article was submitted to Electrochemical Energy Conversion and Storage,

a section of the journal Frontiers in Energy Research

Received: 18 May 2021 Accepted: 30 August 2021 Published: 12 October 2021

Citation:

Din MMU, Häusler M, Fischer SM,

Ratzenböck K, Chamasemani FF, Hanghofer I, Henninge V, Brunner R, Slugovc $C$ and Rettenwander D (2021) Role of Filler Content and Morphology in LLZO/PEO Membranes.

Front. Energy Res. 9:711610. doi: 10.3389/fenrg.2021.711610 content (0, 10, and $20 \mathrm{wt} \%)$ and filler morphology (particles and nanowires) on the electrical and electrochemical properties of the PEO-based composite electrolyte using a wide spectrum of characterization techniques, such as 3D micro-X-ray computed tomography, cross-sectional scanning electron microscopy, X-ray diffraction, and differential scanning calorimetry, impedance spectroscopy, and galvanostatic cycling. The studies reveal that the filler materials are well distributed within the membranes, without any indications for the formation of agglomerates. For $10 \mathrm{wt} \%$ filler, a decrease in the crystallinity compared to PEO was observed, in contrast to 20 wt\% filler showing an increase in crystallinity. Impedance spectroscopic studies on the Li-ion conductivity of the membranes have shown that the change in the Li-ion conductivity is solely related to the change in the crystallinity, rather than to the participation of LLZO as an active transport mediator. The PEO membranes containing $10 \mathrm{wt} \% \mathrm{LLZO}$ have been tested in terms of their rate capability in symmetrical Li cells by galvanostatic cycling. A critical current density of up to $1 \mathrm{~mA} \mathrm{~cm}-2$ at $60^{\circ} \mathrm{C}$ was observed.

Keywords: composite polymer electrolytes, flexible electrolyte membranes, Li garnet, solid-state batteries, high critical current density

\section{INTRODUCTION}

With the increasing sophistication of portable electronic and electric vehicles, there have been continuous developments in energy storage devices, and in the past few years, there have been significant changes in the conventional Li-ion battery system (Li et al., 2020a). However, the low energy density and the Li-dendrite growth in a liquid electrolyte containing LIBs limit its application in electric vehicles and other emerging technologies. Solid-state Li batteries (SSLBs) provide a liquidfree solution that enables new cell architecture for high energy densities and safety (Kamaya et al., 2011). However, to realize this technology, solid-state electrolytes (SSEs) with high Li-ion conductivity, electrochemical stability, structural flexibility, and a wide potential window, are needed. 


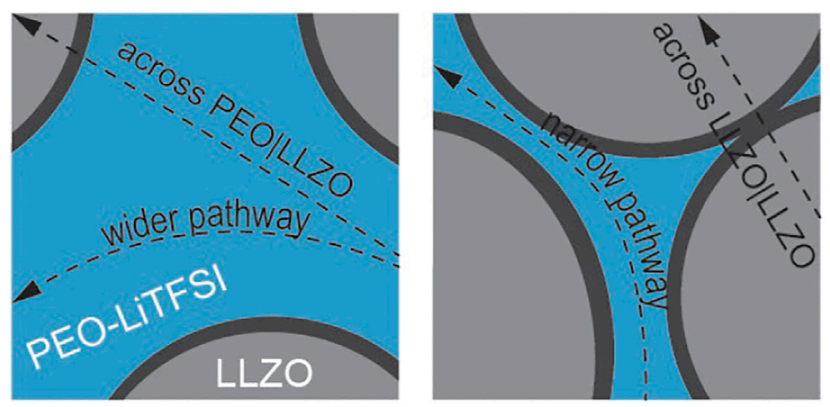

FIGURE 1 | Schematics of Li-ion pathways in PEO-LiTFSI with 10 wt\% (left) and 20 wt\% (right) LLZO filler content. The Li-ion pathways are wider when the filler content is low, thus enabling fast Li-ion transport. At higher filler content, the Li-ion pathways become narrow and the resistance at PEOLiTFSI/LLZO interface is too high, which blocks the Li-ion transport and results in low Li-ion conductivity of the CE.

Poly(ethylene oxide) (PEO) is a widely investigated SSE due to its high flexibility, lightweight, and ease of processability. Furthermore, the presence of ion-solvating ether oxygen group $\left(-\mathrm{CH}_{2}-\mathrm{CH}_{2}-\mathrm{O}-\right)_{\mathrm{n}}$ in its backbone makes it an excellent solvating medium for a range of conducting salts such as $\mathrm{LiClO}_{4}$ and $\mathrm{LiCF}_{3} \mathrm{SO}_{3}$ (Baril et al., 1997). However, poly(ethylene oxide) containing the conducting salts suffers from a serious issue of poor ionic conductivity at room temperature $\left(\sim 10^{-6} \mathrm{~S} \mathrm{~cm}^{-1}\right)$ and mechanical instabilities (Langer et al., 2016; Long et al., 2016).

To improve ionic conductivity, various types of filler materials, such as $\mathrm{SiO}_{2}$ (Lin et al., 2016) and $\mathrm{TiO}_{2}$ (Lin et al., 2005), were added into PEO to improve the overall conductivity by lowering the crystallinity of the polymer (Tan et al., 2018). Thereafter, Liion conductive ceramic fillers have been extensively studied due to the promise to deliver a further improvement in $\mathrm{Li}$-ion conductivity by contributing to the long-range Li-ion transport as well as an improvement in the mechanical and electrochemical characteristics (Liu et al., 2015; Liu et al., 2017; Bonizzoni et al., 2019; Zaman et al., 2019). Among Li-ion conducting fillers, $\mathrm{Li}_{7} \mathrm{La}_{3} \mathrm{Zr}_{2} \mathrm{O}_{12}$ (LLZO) garnet $\left(\sigma \leq 10^{-3} \mathrm{~S} \mathrm{~cm}^{-1}\right.$ ) (Wu et al., 2017) has been extensively explored due to its high Li-ion conductivity, excellent stability against Li metal electrode, and wide potential window (Murugan et al., 2007; Rettenwander et al., 2016).

Chen et al. (2017) have reported that a $7.5 \mathrm{wt} \%$ LLZO filler acts as an active ion conductor in an LLZO/PEO composite electrolyte (CE), improving the overall $\mathrm{Li}$-ion conductivity to a value of $5.5 \times$ $10^{-4} \mathrm{~S} \mathrm{~cm}^{-1}$. In contrast, other groups have reported that this increase in the Li-ion conductivity by adding small weight percentages of LLZO is attributed to the slight increase in the amorphous PEO phase (similar to nonconducting fillers) (Zagórski et al., (2019). For LLZO content larger than 7.5 wt\% a decrease in the Li-ion conductivity is reported by Chen et al. (2017) and Karthik and Murugan (2019), indicating that the embedded LLZO particles do not contribute to long-range transport. Zagórski et al. (2019) investigated the CE containing both multiphase and phase pure LLZO fillers and revealed that the ionic conductivity of the $\mathrm{CE}$ was independent of the purity of the LLZO filler, as similar Li-ion conductivities were reported for both cases. This observation additionally hints toward the conclusion that LLZO filler may not participate as an active ion conductor in the CE.

The passive role of LLZO content in the CE could be related to the high interfacial resistances between LLZO and PEO (Brogioli et al., 2019; Keller et al., 2017), which limits the Li-ion mobility (Figure 1). For example, Gupta and Sakamoto (2019) reported a large interfacial resistance of $96 \mathrm{k} \Omega \mathrm{cm}^{2}$ for PEO-LiTFSI/LLTZO. The large interface resistance is the outcome of LLZO surface impurities and/or surface defects. By optimizing the LLZTO surface, the interface resistance was reduced to $180 \Omega \mathrm{cm}^{2}$ at $30{ }^{\circ} \mathrm{C}$. They hypothesized that interfacial resistance below $100 \Omega \mathrm{cm}^{2}$ is a prerequisite to enable $\mathrm{Li}$-ion transport across the PEO/LLZTO interface (Gupta and Sakamoto, 2019). The $\mathrm{PEO} / \mathrm{LLZO}$ interface in CEs, however, is complex and differs invariably from that of the PEO/LLZO pellet interface. Therefore, tuning such an interface with similar approaches to achieve low resistance values is difficult. A relatively low resistance of $500 \Omega \mathrm{cm}^{2}$ at $20 \mathrm{C}$ for the PEO-LiTFSI/LLZO pellet interface was reported by Kuhnert et al. (2020) by a facile surface treatment of LLZTO. However, the interface resistance is still too high to promote Li-ion migration throughout the whole electrolyte. Hence, the Li solely migrates through PEO (Figure 1) (Chen et al., 2017; Zheng and Hu, 2018; Karthik and Murugan, 2019; Li et al., 2020b; Nguyen et al., 2020).

It is suggested that an increase in the filler concentrations to a value larger than $50 \mathrm{wt} \%$ that allows a percolating network, will allow fast ion-conducting tracks that enable a high Li-ion conductivity value similar to the dense sintered LLZO (Zheng et al., 2016; Chen et al., 2018). In order to make a percolating network, several groups focused on the design of membranes composed of LLZO nanowires. Those studies reported a significant increase in the room temperature Li-ion conductivity by two orders of magnitude by adding only 5 to 10 wt \% of LLZO (Yang et al., 2017; Wan et al., 2019). The Li-ion conductivity enhancement is ascribed to the high ionic conductivity of LLZO nanowires and the uniform distribution of LLZO nanowires in the polymer matrix that establishes a continuous network for efficient Li-ion transport (Wan et al., 2019). However, the morphology of the CE membranes does not reveal any clear evidence for the uniform distribution of LLZO nanowires or the formation of the percolating network. The nanofibers are also generally sintered at lower temperatures to retain the fiber morphology; therefore, it is unlikely that the LLZO nanowires are highly Li-ion conductive.

To better understand the role of filler morphology (particles vs. nanowires) in PEO on the $\mathrm{Li}$-ion transport, we synthesized $\mathrm{Li}_{6.4} \mathrm{Ga}_{0.2} \mathrm{La}_{3} \mathrm{Zr}_{2} \mathrm{O}_{12} \quad$ (pLLZO) particles and $\mathrm{Li}_{7} \mathrm{La}_{3} \mathrm{Zr}_{2} \mathrm{O}_{12}$ (nwLLZO) by ceramic sintering and electrospinning, respectively. Thereafter, PEO membranes with $10 \mathrm{wt} \%$ and $20 \mathrm{wt} \%$ filler content were prepared by a solution cast technique. The filler morphologies, distribution, and connectivity in the polymer matrix were investigated by means of X-ray tomography and SEM on cross sections prepared by ion slicing. The Li-ion conductivity of the membranes was studied by using impedance spectroscopy. Moreover, the rate capability of 
the membranes was tested in symmetrical $\mathrm{Li}$ cells using galvanostatic cycling.

We found that the filler distribution was homogeneous within the PEO matrix with $10 \mathrm{wt} \%$ and $20 \mathrm{wt} \%$ pLLZO and nwLLZO, and no filler agglomerations were observed. The highest Li-ion conductivity of $1.36 \times 10^{-5} \mathrm{~S} \mathrm{~cm}^{-1}$ at $25^{\circ} \mathrm{C}$ was achieved with $10 \mathrm{wt} \%$ pLLZO filler content, which may be related to the disruption in polymer crystallization and the resulting increase in disorder of Li-ion local environments within the PEO matrix. A further increase in the filler concentration leads to a decrease in the Li-ion conductivity. Based on our results, a high filler concentration does not lead to an improvement in CEs. We hypothesize that this is related to the following two reasons: 1) the higher filler content may arrest the segmental motion of polymer chains and 2) the narrowing of active Li-ion conduction pathways by reducing the $\mathrm{PEO}$ portion in the $\mathrm{CE}$. The homogeneous distribution of ceramic filler allowed reversible cycling of $\mathrm{Li}$ with rates as high as $1 \mathrm{~mA} \mathrm{~cm}^{-2}$ at $60^{\circ} \mathrm{C}$ for the $\mathrm{PEO}$ membranes containing $10 \mathrm{wt} \%$ nwLLZO.

\section{EXPERIMENTAL}

\section{LLZO Particles}

Ga-doped LLZO was prepared by a solid-state reaction route. Briefly, $\mathrm{Li}_{2} \mathrm{CO}_{3}$ (99\%; Alfa Aesar), $\mathrm{La}_{2} \mathrm{O}_{3}$ (99.9\%; Alfa Aesar), $\mathrm{ZrO}_{2}$ (99\%; Sigma-Aldrich), and $\mathrm{Ga}_{2} \mathrm{O}_{3}$ (99.999\%; Alfa Aesar) were used as reaction precursors for the preparation of pLLZO. $\mathrm{La}_{2} \mathrm{O}_{3}$ was predried at $900^{\circ} \mathrm{C}$ for $48 \mathrm{~h}$ to remove the traces of moisture. $10 \mathrm{wt} \%$ excess $\mathrm{Li}$ was used to avoid $\mathrm{Li}$ loss during sintering. The reaction precursors were hand-mixed together and calcined at $850^{\circ} \mathrm{C}$ for $6 \mathrm{~h}$. The obtained powder was later ballmilled using zirconia balls in isopropanol at an rpm of 800 for $1 \mathrm{~h}$ in a Fritsch ball mill (Planetary Mono Mill PULVERISETTE 6). The milling time was set for $5 \mathrm{~min}$ and the pause time was $10 \mathrm{~min}$ with 11 repetitions. The powder recovered after drying the traces of isopropanol was pressed into thin pellets of $10 \mathrm{~mm}$ diameter. The pellets were transferred into a platinum crucible and sintered at $1,150^{\circ} \mathrm{C}$ for $6 \mathrm{~h}$ in a muffle furnace. To avoid any Li loss during high-temperature sintering, the pellets were covered with a sufficient amount of sacrificial powder. The sintered pellets were polished with sandpapers and stored in an inert atmosphere. The phase purity was confirmed, and the Li-ion conductivity of the pellets was measured.

To prepare the pLLZO powder for the polymer membranes, high Li-ion conductive pellets were crushed and ball-milled following a similar procedure as described above. The recovered powder was dried and calcined at $450^{\circ} \mathrm{C}$ for $3 \mathrm{~h}$ in a furnace placed inside an Ar-filled glove box $\left(\mathrm{O}_{2}\right.$ and $\mathrm{H}_{2} \mathrm{O}<$ $0.1 \mathrm{ppm})$.

\section{LLZO Nanowires}

Dopant-free cubic nanostructured LZO garnet was prepared at room temperature by the electrospinning method. $\mathrm{LiNO}_{3}$ (99\%; Alfa Aesar), $\mathrm{La}\left(\mathrm{NO}_{3}\right)_{3} \cdot 6 \mathrm{H}_{2} \mathrm{O}\left(99 \%\right.$; Fluka), and $\mathrm{ZrO}\left(\mathrm{NO}_{3}\right)_{2} \cdot 6 \mathrm{H}_{2} \mathrm{O}$ (99.9\%; SigmaAldrich) in stoichiometric amounts were dissolved in dimethylformamide to prepare a sol precursor. $10 \mathrm{wt} \%$ excess $\mathrm{Li}$ was used to compensate Li loss during the calcination process. A small amount of acetic acid was added as a chelating agent that prevents the hydrolysis of alkoxides. $8.8 \mathrm{wt} \%$ polyvinylpyrrolidone (PVP) (molecular weight, 1,300,000; Sigma-Aldrich) was added to the sol precursor to prepare the electrospinning solution. An electrospinning setup with a horizontally connected pump and drum collector was used for this process. The solution was filled in a syringe with a needle gauge size $20 \mathrm{G}$, and the feed rate was controlled with the pump $\left(0.45 \mathrm{ml} \mathrm{h}^{-1}\right)$. During electrospinning, a high voltage of $16.1 \mathrm{kV}$ was applied between the needle and the drum collector. The drum collector was connected to the ground. The distance between the needle and the drum collector was $12 \mathrm{~cm}$. The drum collector was covered with aluminum foil to collect the as-spun nanofibers. The rotation speed of the drum was controlled (4 rpm), and the relative humidity of the electrospinning chamber was maintained at $10 \%$. After electrospinning, the as-spun nanofibers were peeled off from the aluminum foil and then calcinated at $700^{\circ} \mathrm{C}$ for $2 \mathrm{~h}$ in the air to obtain the nwLLZO.

\section{Membranes}

Polyethylene oxide (PEO) with a molecular weight of 400,000 (SigmaAldrich) and lithium bis(trifluromethanesulfonyl)imide (LiTFSI, Sigma-Aldrich) were used as polymer and conducting salt, respectively. The molar ratio of the monomer units of PEO to the number of Li-ions was kept constant at 15:1. PEO and LiTFSI were dissolved in acetonitrile under magnetic stirring at $60^{\circ} \mathrm{C}$ for $6 \mathrm{~h}$ to obtain a clear viscous solution. Appropriate amounts of pLLZO and nwLLZO were dispersed in acetonitrile by ultrasonication. The two solutions were mixed together to form homogeneous slurries under magnetic stirring for $3 \mathrm{~h}$ at $25^{\circ} \mathrm{C}$. The slurries were cast on a flat Teflon plate and left for room drying. The dried tapes were vacuum-dried at $50^{\circ} \mathrm{C}$ for $24 \mathrm{~h}$ and transferred into a glove box to avoid any contamination from the atmosphere. Four different samples were prepared based on the filler content and filler morphology. The samples containing 10 and $20 \mathrm{wt} \%$ pLLZO are designated as PEO: pLLZO-10 and PEO:pLLZO-20 while the samples containing 10 and $20 \mathrm{wt} \%$ LLZO are designated as PEO:nwLLZO-10 and PEO: nwLLZO-20. Pristine PEO:LiTFSI was also prepared for comparison and is designated as PEO. The thickness of the membranes ranged from 50 to $70 \mu \mathrm{m}$.

\section{Characterization}

The phase purity of pLLZO, nwLLZO, and polymeric membranes was confirmed by X-ray diffraction (Rigaku X-ray diffractometer with $\mathrm{Cu}$ / ka radiation $(\lambda=0.15418 \mathrm{~nm})$ and a $2 \theta$ range $\left.=10-90^{\circ}\right)$. The size distribution of pLLZO was measured by a particle size analyzer (CILAS particle size analyzer). A scanning electron microscope (Zeiss EVO MA 25) was used for material imaging. 3D micro-Xray computed tomography ( $\mu-\mathrm{XCT}$ ) was utilized to provide the $3 \mathrm{D}$ pLLZO particle distribution in the two different polymer membranes. Therefore, we used a nanotom from General Electrics (GE). The voxel size was $690 \mathrm{~nm}$ and the selected representative volume of interest (VOI) for the analysis was $429 \times 38 \times 438 \mu \mathrm{m}^{3}$. The $\mu$-XCT data were preprocessed by applying a median filter to improve the image quality. Subsequently, a histogram-based global thresholding method was used to segment the pLLZO particles from the polymer matrix based on the gray values in the image data (Vorauer et al., 2020). Finally, a 

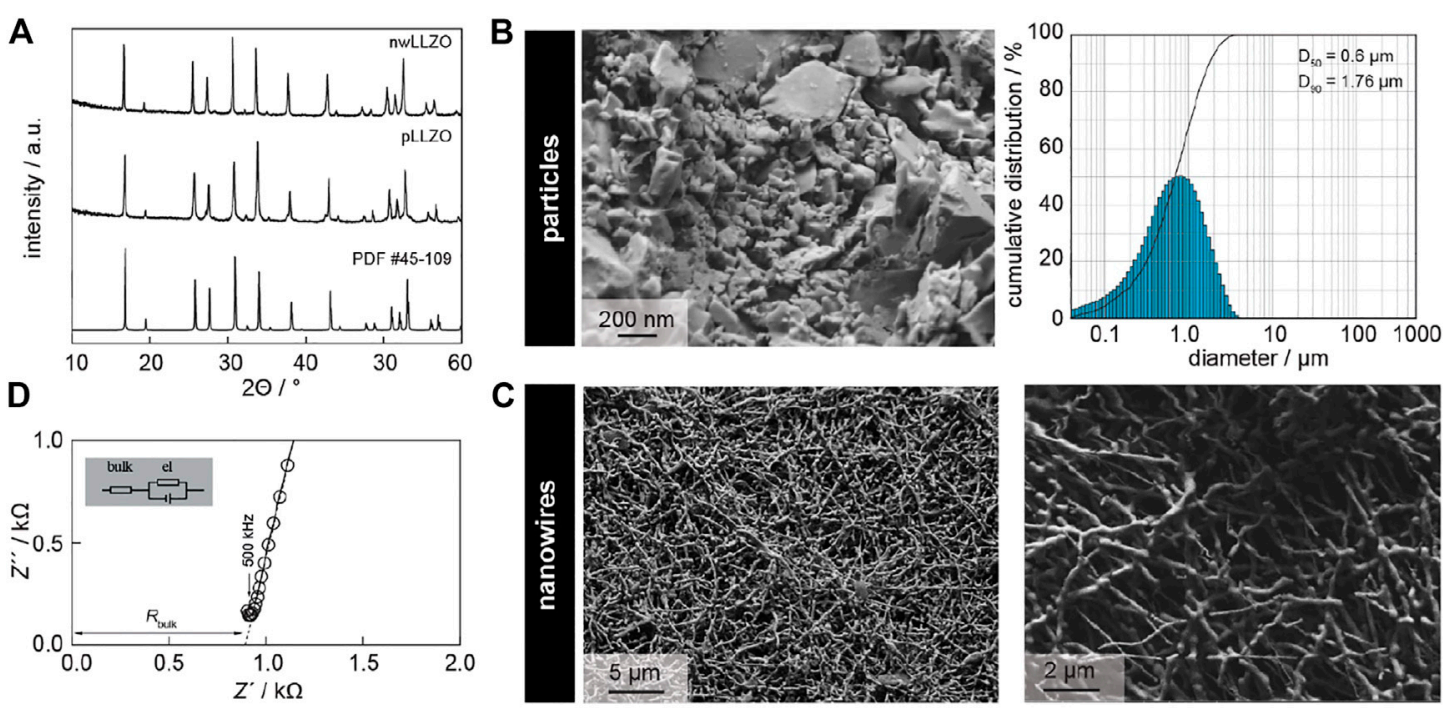

FIGURE 2 | (A) X-ray diffraction patterns of pLLZO and nwLLZO along with the JCPDS of cubic garnet. (B) SEM image and particle size distribution of pLLZO. (C) SEM images of the as-prepared nwLLZO (left) and calcined nwLLZO (right). (D) Nyquist plot of pLLZO and inset in (D) is the equivalent circuit diagram used for simulation.

watershed algorithm was used to evaluate the equivalent diameter of the particles. The segmented image data were visualized by using Avizo. Further, we used, in comparison with the $\mu$-XCT, a GeminiSEM 450 from Zeiss to investigate the particle distribution on smaller length scales in the cross section. Therefore, to prepare the cross section, we used an ionslicer Hitachi IM4000. Thermal properties of polymer membranes were investigated by DSC (Netzsch STA 449 Jupiter) in a temperature range of $-60^{\circ} \mathrm{C}-100^{\circ} \mathrm{C}$ with a heating rate of $10^{\circ} \mathrm{C} / \mathrm{min}$. Electrochemical impedance spectroscopy (EIS) of polymer membranes was carried out using battery tester (BioLogic VMP3). Au was used as a blocking electrode to perform the EIS of pLLZO. For the EIS tests, polymer membranes were sandwiched between the two stainless steel discs and assembled in Swagelok cells. GCPL measurements were performed for the Li symmetric cells using a Solartron battery cycler. Symmetrical cells with disc-shaped $\mathrm{Li}$ electrodes and $\mathrm{CE}$ were assembled into Swagelok-type cells in an argon-filled glove box $\left(\mathrm{O}_{2}\right.$ and $\mathrm{H}_{2} \mathrm{O}<$ $0.1 \mathrm{ppm}$ ) at room temperature.

\section{RESULTS AND DISCUSSION}

\section{Ceramic Filler}

$\mathrm{X}$-ray diffraction patterns of pLLZO and nwLLZO are shown in Figure 2A. The comparison of the occurring reflections of the samples with the reference pattern shows a good agreement for both pLLZO and nwLLZO, indicating the successful synthesis of cubic LLZO. For nwLLZO, however, an additional reflex at $28^{\circ} 2 \theta$ is observed. This reflex can be assigned to pyrochlore $\left(\mathrm{La}_{2} \mathrm{Zr}_{2} \mathrm{O}_{7}\right)$, a phase that typically forms during Li loss at high-temperature processing (Fu et al., 2016). The loss of $\mathrm{Li}$ is considerably faster for nwLLZO due to their large surface area compared to pLLZO. A lowering of the sintering temperature or a shorter dwelling time has been reported to result in an incomplete reaction of the precursor salts (Yang et al., 2017). Despite the presence of the intermediate pyrochlore phase and the absence of a doping element, nwLLZO was present in the cubic phase, which is related to the high surface energy of nanoparticles due to their high curvatures. In Figure 2B, the particle morphology of pLLZO and the particle size distribution is shown. The particles show irregular shapes as seen from the SEM image, and the particle size distribution shown in Figure 2B reveals that the particle diameters for $\mathrm{D}_{50}$ and $\mathrm{D}_{90}$ are 0.6 and $1.76 \mu \mathrm{m}$, respectively. The SEM images of nwLLZO are shown in Figure 2. A large network of wires is clearly visible. The further magnification reveals individual wires forming cross-linked 3D nanofiber networks. The wire diameters vary from 100 to $300 \mathrm{~nm}$ compared to the dimensions of nanowires mentioned in the literature (Wan et al., 2019). The nwLLZO usually measures several micrometers and also many millimeters in length. However, the length of individual fibers cannot be clearly determined as the fibers formed an interwelded 3D network structure during the calcination process. The Li-ion conductivity of the pLLZO (as a sintered pellet) was studied via impedance spectroscopy. In Figure 2D, the corresponding complex plane plot is shown revealing two electrical relaxation processes: a high-frequency semicircle and a spike towards low frequencies The spectrum can be fitted by a resistance $(R)$ serial to an additional $\mathrm{R}$ in parallel to a constant phase element $(R / \mathrm{CPE}$; see inset Figure 2). The $R / C P E$ element improves the fit due to the nonideality of the blocking electrode but has no physical meaning. The corresponding total Li-ion conductivity of pLLZO is given by $\sigma_{\mathrm{pLLZO}}=R_{\mathrm{pLLZO}}{ }^{-1} A^{-1} d$, with $A$ being the electrode area and $d$ being the thickness. The calculated total Liion conductivity of $3.19 \times 10^{-4} \mathrm{~S} \mathrm{~cm}^{-1}$ is similar to values reported in the literature (Wolfenstine et al., 2012). 


\section{Membranes}

In Figure 3, the digital images of the respective CEs containing 10 and $20 \mathrm{wt} \%$ of pLLZO and nwLLZO as well as $30.1 \mathrm{wt} \%$ LiTFSI in PEO are shown. The CEs are very thin $(50-70 \mu \mathrm{m})$ as well as smooth and highly flexible. The lower membrane thickness resulting in higher flexibility is the important prerequisite for a viable CE.

\section{Nomweg:in University of

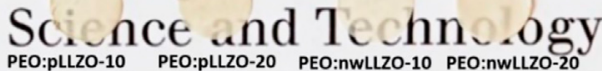

FIGURE 3 | Digital images of the as-prepared CE membranes.
Figures $4 A, B$ depict the segmented pLLZO particles and $3 \mathrm{D}$ particle size distribution for the representative VOI with $10 \mathrm{wt} \%$ and $20 \mathrm{wt} \%$ filler, respectively. It can be observed that the pLLZO particles are uniformly distributed in the polymer matrix containing pLLZO-10 and pLLZO-20.

A higher particle concentration was observed for pLLZO20 than for pLLZO-10. The extracted mean diameter, median, and standard deviation as well as the maximum and minimum diameter are similar for pLLZO-10 and pLLZO-20, respectively. Further, to prove the homogeneity of the CEs, cross-sectional SEM images were recorded. From Figures 4C, $\mathbf{D}$, it is seen that the pLLZO and nwLLZO are homogeneously distributed in the polymer matrix; no indication of the formation of filler agglomerates is found. When the concentration of filler is $20 \mathrm{wt} \%$, the filler particles are very closely packed in the polymer matrix as clearly seen in Figures
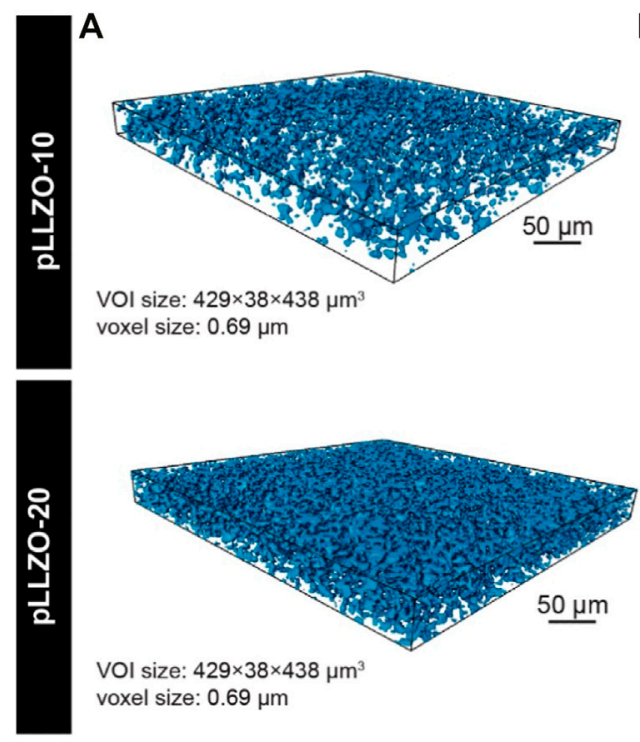

VOI size: $429 \times 38 \times 438 \mu \mathrm{m}^{3}$

voxel size: $0.69 \mu \mathrm{m}$
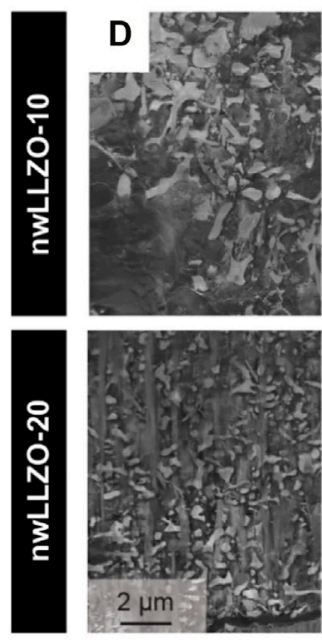
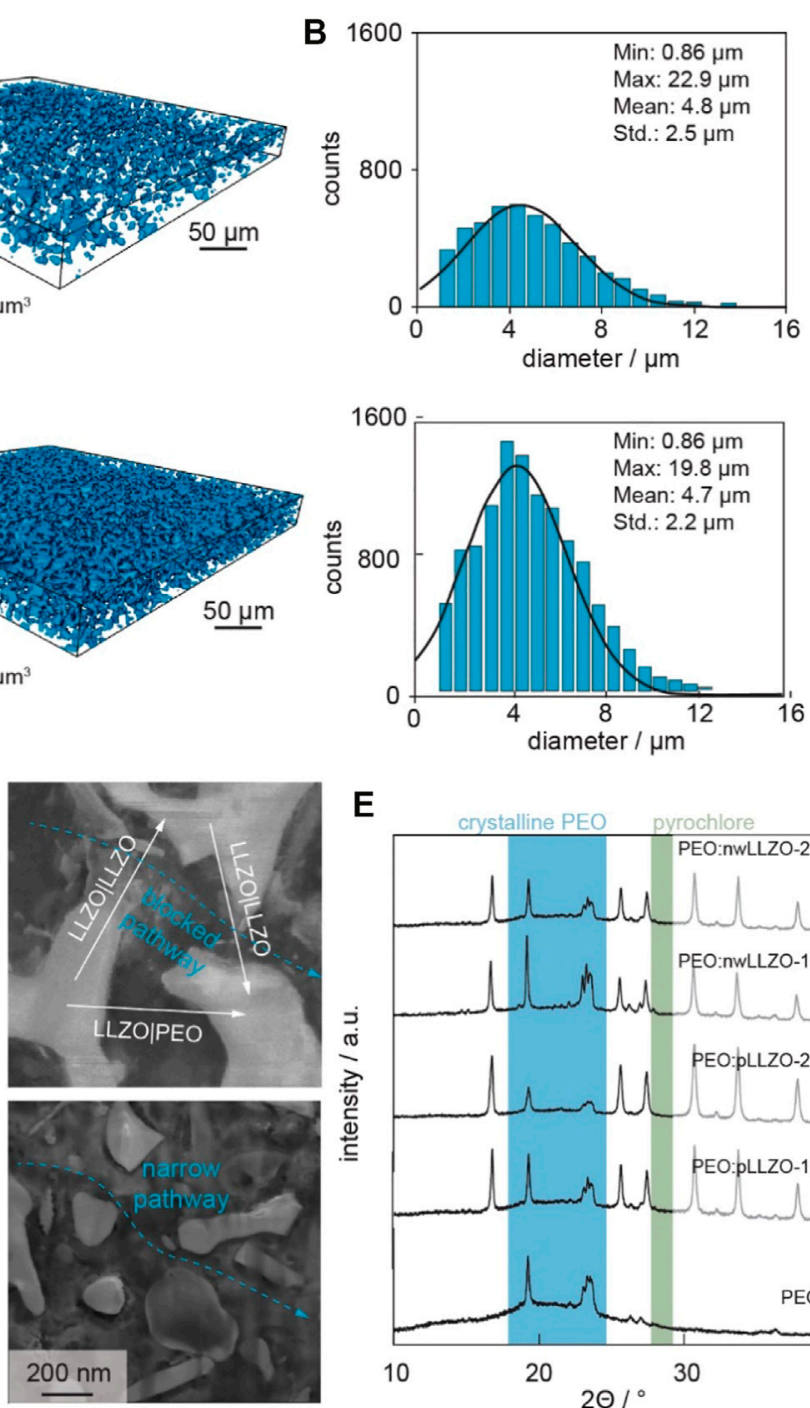

E

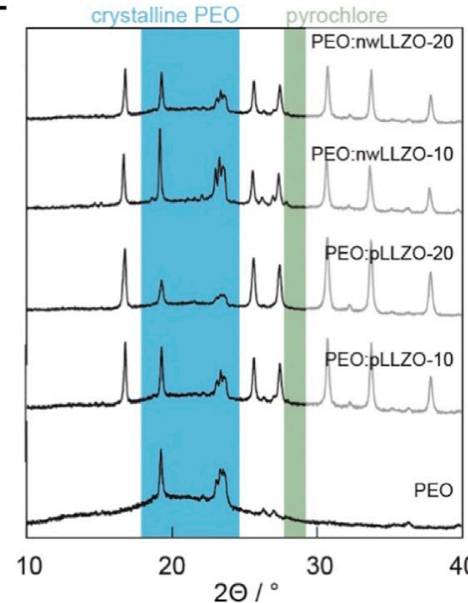

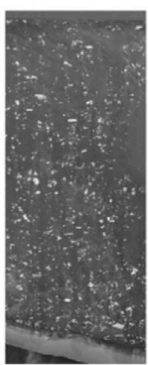
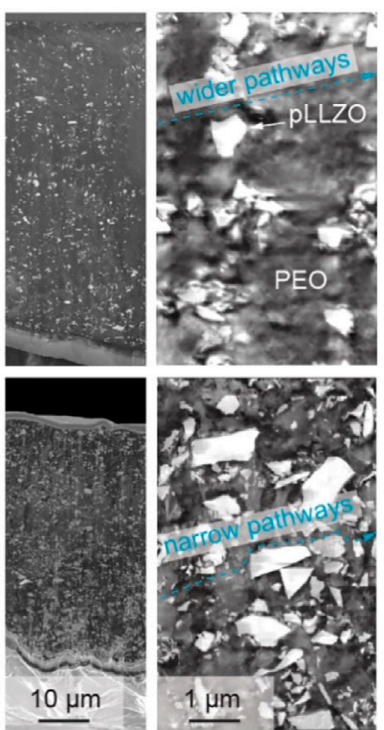

$\mathbf{F}$

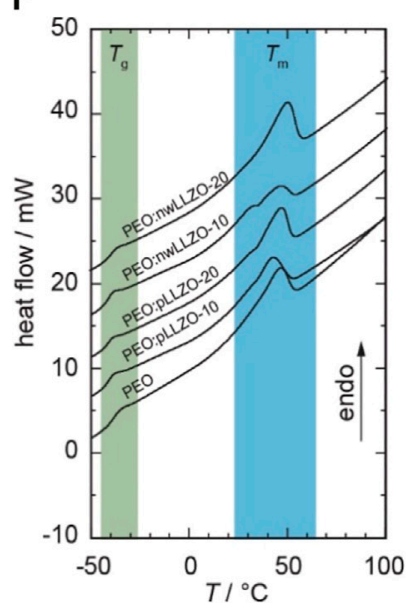

FIGURE 4 | (A) 3D $\mu$-XCT analysis and (B) image analyzed by pLLZO 3D filler distribution in the PEO matrix. (C) Cross-sectional SEM of PEO containing 10 and 20 wt\% pLLZO. (D) Cross-sectional SEM of PEO containing 10 and 20 wt\% nwLLZO. (E) X-ray diffraction patterns and (F) DSC for the respective CE membranes. 
TABLE 1 | Results from DSC, EIS, and galvanostatic cycling.

\begin{tabular}{|c|c|c|c|c|c|c|c|c|}
\hline \multirow[t]{2}{*}{ Membrane } & \multirow[t]{2}{*}{$T_{\mathrm{g}}\left({ }^{\circ} \mathrm{C}\right)$} & \multirow[t]{2}{*}{$T_{m}\left({ }^{\circ} \mathrm{C}\right)$} & \multirow[t]{2}{*}{$\Delta H_{\mathrm{m}}\left(\mathrm{J} \mathrm{g}^{-1}\right)$} & \multirow[t]{2}{*}{$\chi_{c}(\%)$} & \multirow[t]{2}{*}{$\sigma_{\mathrm{RT}}\left(\mathrm{S} \mathrm{cm}^{-1}\right)$} & \multicolumn{2}{|c|}{$E_{\mathrm{a}}(\mathrm{eV})$} & \multirow[t]{2}{*}{$\operatorname{CCD}\left(\mathrm{mA} \mathrm{cm} \mathrm{cm}^{-2}\right)$} \\
\hline & & & & & & $20-50^{\circ} \mathrm{C}$ & $50-100^{\circ} \mathrm{C}$ & \\
\hline PEO (with LiTSFI) & -44 & 45 & 23 & 16 & $0.78 \times 10^{-5}$ & - & - & - \\
\hline PEO:pLLZO-10 & -46 & 42 & 22 & 15 & $1.36 \times 10^{-5}$ & 0.83 & 0.40 & 0.5 \\
\hline PEO:pLLZO-20 & -39 & 46 & 28 & 20 & $1.04 \times 10^{-5}$ & 0.79 & 0.48 & - \\
\hline PEO:nwLLZO-10 & -45 & 45 & 18 & 13 & $0.93 \times 10^{-5}$ & 0.90 & 0.45 & 1.0 \\
\hline PEO:nwLLZO-20 & -38 & 50 & 30 & 21 & $0.43 \times 10^{-5}$ & 0.81 & 0.45 & - \\
\hline
\end{tabular}

$\mathrm{T}_{\mathrm{g}}$, glass transition temperature; $\mathrm{T}_{\mathrm{m}}$, melting temperature; $\Delta \mathrm{H}_{m}$, melting enthalpy; $\chi_{\mathbf{c}}$, degree of crystallinity; $\mathrm{E}_{a}$, activation energy; $\sigma_{R T}, L i$-ion conductivity at room temperature; and CCD, critical current density

4C,D. This in turn could result in blocking the Li-ion migration through the polymer matrix by decreasing the free volume for the segmental motion of PEO chains. The PXRD pattern of CE is shown in Figure 4. The sharp reflexes in the lower $2 \theta$ region of the filler-free membrane indicate the crystalline nature of PEO (Cheng et al., 2017). The crystallinity of PEO alters by adding the filler, which is beneficial for high chain mobility, hence for high Li-ion conductivities within the polymer matrix.

To study the thermal behavior and to quantify the crystallinity of PEO in PEO, PEO:pLLZO-10, PEO:pLLZO-20, PEO:nwLLZO-10, and PEO:nwLLZO-20, DSC measurements were performed. The glass transition temperature $\left(T_{g}\right)$ of polymers indicates the temperature above which the polymer molecules are free to move. Lowering the $T_{g}$ of PEO to improve the polymer chain mobility is, therefore, one of the effective ways to increase the Li-ion conductivity (Xue et al., 2015). The DSC results show that the glass temperature $T_{g}$ of CE slightly decreased after adding $10 \mathrm{wt} \%$ pLLZO filler to the polymer host as depicted in Figure 3F. The $T_{g}$ of PEO:pLLZO-10 and PEO:nwLLZO- 10 was $-46^{\circ} \mathrm{C}$ and $-45^{\circ} \mathrm{C}$, respectively, compared to $T_{g}$ of $-44^{\circ} \mathrm{C}$ for PEO (Balo et al., 2017). However, the $T_{g}$ of PEO:pLLZO20 and PEO:nwLLZO-20 was $-39^{\circ} \mathrm{C}$ and $-38^{\circ} \mathrm{C}$, respectively. The melting temperatures are reduced to $42^{\circ} \mathrm{C}$ and $45^{\circ} \mathrm{C}$ for PEO:pLLZO10 and PEO:nwLLZO-10, respectively, in comparison with $46^{\circ} \mathrm{C}$ and $50^{\circ} \mathrm{C}$ for PEO:pLLZO-20 and PEO:nwLLZO-20. The degree of crystallinity was calculated using $\chi_{\mathrm{c}}=\Delta H_{\mathrm{m}} /\left(\Delta \mathrm{H}_{\mathrm{c}} \times f_{\mathrm{PEO}}\right) \times 100$, where $\Delta H_{\mathrm{m}}$ is the melting enthalpy, $\Delta H_{\mathrm{c}}$ is the melting enthalpy of crystalline PEO (203 J g ${ }^{-1}$ ) (Wu et al., 2011), and $f_{P E O}$ is the PEO mass percentage in the CEs. The degrees of crystallinity for PEO, PEO: pLLZO-10, PEO:pLLZO-20, PEO:nwLLZO-10, and PEO:nwLLZO20 were $16,15,20,13$, and $21 \%$, respectively. These results indicate that PEO:pLLZO-10 and PEO:nwLLZO-10 should have increased mobility of polymer chains and improved Li-ion conductivity compared to the membranes with higher filler content (in case that LLZO does not take place actively in the overall Li-ion transport). The parameters deduced from the DSC are summarized in Table 1.

\section{Electrochemical Performance of Membranes}

\section{Ionic Conductivity}

To study the influence of the filler morphology and concentration, the Li-ion conductivity of the CEs was determined by electrochemical impedance spectroscopy. Representative Nyquist plots of the membranes measured at $25^{\circ} \mathrm{C}$ and the associated equivalent circuit are shown in Figure 5. The impedance spectra show an onset of a semicircle towards high frequencies well separated from the electrode polarization towards low frequencies. The spectra can be well fitted by two serially connected resistance elements with CPE elements in parallel (see inset Figure 5A), whereby the $\mathrm{R} / \mathrm{CPE}$ element for the electrode has no physical meaning. The Liion conductivity of PEO, PEO:pLLZO-10, and PEO:pLLZO-20 at $25^{\circ} \mathrm{C}$ was found to be $0.78 \times 10^{-5} \mathrm{~S} \mathrm{~cm}^{-1}, 1.36 \times 10^{-5} \mathrm{~S} \mathrm{~cm}^{-1}$, and $1.04 \times 10^{-5} \mathrm{~S} \mathrm{~cm}^{-1}$, respectively, while Li-ion conductivity of PEO:nwLLZO-10 and PEO:nwLLZO-20 was $0.93 \times$ $10^{-5} \mathrm{~S} \mathrm{~cm}^{-1}$ and $0.43 \times 10^{-5} \mathrm{~S} \mathrm{~cm}^{-1}$, respectively.

The lower Li-ion conductivity of PEO containing a higher amount of LLZO indicates that LLZO does not actively take part in the long-range Li-ion transport by providing fast ion-transport tracks, which is related to the high interface resistance between PEO and LLZO (Brogioli et al., 2019; Kuhnert et al., 2020). Hence, any further addition of LLZO filler leads to a narrowing or blocking of the Li-ion migration channels by restricted chain mobility (Zheng and $\mathrm{Hu}, 2018$; Schneider, 2017), which reduces the Li-ion conductivity (Chen et al., 2018). As evident from Figures 4C, D, there are no particle agglomerations within the membranes, which could allow Li-ion conduction through a percolating ceramic network. Based on our understanding, even when Li-ion transport would take place in well interconnected LLZO particles, no improvement in terms of the overall Li-ion conductivity can be expected. For example, compressed LLZO powders have conductivities of more than two to three orders of magnitude lower $\left(\sim 10^{-6} \mathrm{~S} \mathrm{~cm}^{-1}\right)$ compared to the sintered LLZO pellets $\left(\sim 10^{-3} \mathrm{~S} \mathrm{~cm}^{-1}\right)$ (Zagórski et al., 2019). This low Li-ion conductivity in a percolating network is related to the high interface resistance between the loosely bonded ceramic particles. Hence, this value can be considered as the maximum value that can be achieved in CE, which is similar to the conductivity achieved for PEO without adding any filler.

To study the temperature dependency of the Li-ion conductivity, impedance was recorded from 20 to $100^{\circ} \mathrm{C}$ in a frequency range of $1 \mathrm{MHz}$ to $1 \mathrm{~Hz}$. In Figure 5, the conductivity values for temperature from 20 to $60^{\circ} \mathrm{C}$ are shown for comparison. The Arrhenius representation for the $\mathrm{Li}$-ion conductivities of the membranes in the temperature range of $20^{\circ} \mathrm{C}$ to $100^{\circ} \mathrm{C}$ is shown in Figure 5. The activation energies of the CEs were calculated according to $\sigma=\mathrm{A} \exp$ 

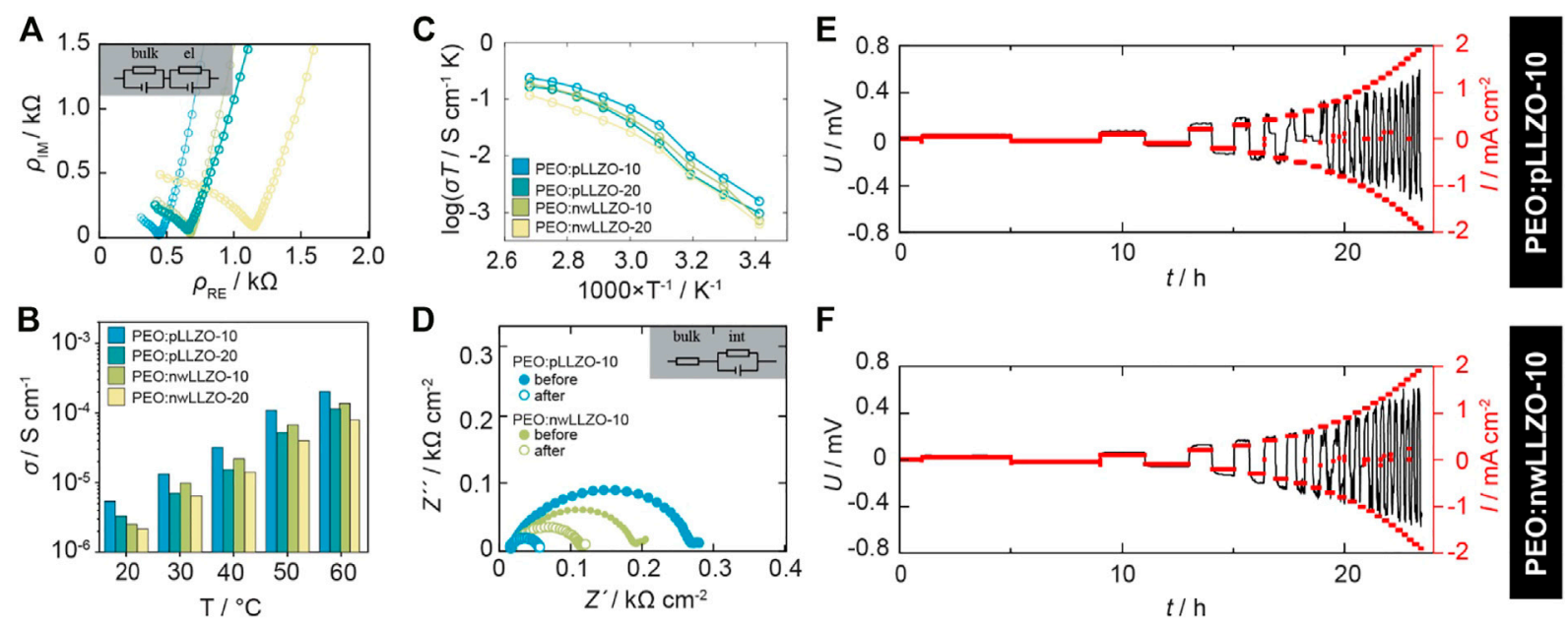

FIGURE 5 | (A) Nyquist plots of PEO:pLLZO-10, PEO:pLLZO-20, PEO:nwLLZO-10, and PEO:nwLLZO-20 at 25 $5^{\circ}$, and inset represents the corresponding equivalent circuit. (B) Temperature-dependent Li-ion conductivity of PEO:pLLZO-10, PEO:pLLZO-20, PEO:nwLLZO-10, and PEO:nwLLZO-20. (C) Arrhenius plots for PEO:pLLZO-10, PEO:pLLZO-20, PEO:nwLLZO-10, and PEO:nwLLZO-20. (D) Impedance spectra of PEO:pLLZO-10 and PEO:nwLLZO-10 before and after short circuit during galvanostatic cycling, and inset in (D) is the equivalent circuit adopted for simulation. Time-dependent current and voltage profiles of Li symmetric cells consisting of (E) PEO:pLLZO-10 and (F) PEO:nwLLZO-10 at 60 6 .

$\left(-E_{\mathrm{a}} / \mathrm{k} T\right)$, where $\sigma$ is the ionic conductivity, $A$ is the pre-exponential constant, $E_{\mathrm{a}}$ is the activation energy for Li-ion conduction, $k$ is the Boltzmann constant, and $T$ is the absolute temperature. In the lowtemperature range $\left(20-50^{\circ} \mathrm{C}\right)$, the sluggish segmental motion of polymeric chains and lower viscosity of the polymer lead to a strong temperature dependency of the Li-ion conductivity with an $E_{\mathrm{a}}$ in the range $0.79-0.90 \mathrm{eV}$. Toward high temperatures, the PEO starts to melt and the conductivity of the CE increases dramatically due to the crystalline-amorphous phase transition (Ngai et al., 2016). Afterward, in the region $\left(50-100^{\circ} \mathrm{C}\right)$ when the polymer is in the melt phase, the mobility of polymeric chains increases dramatically, thus resulting in excellent ionic transportation with $E_{a}$ in the range $0.40-0.48 \mathrm{eV}$. The calculated activation energy values are in close agreement with those reported in the literature (Choi et al., 2015; Chen et al., 2017).

Overall, these results suggest that the key to high Li-ion conductivities in $\mathrm{CE}$ membranes is the disruption in crystallinity of the membranes rather than the filler content. Further research is needed to reduce the crystallinity of PEO membranes by using modifiers or plasticizers to achieve high ionic conductivities at room temperature. In addition, new strategies need to be designed to decrease the interface resistance between PEO and LLZO below $100 \Omega \mathrm{cm}^{2}$ to make LLZO an active component in CEs for the development of flexible $\mathrm{CE}$ with superior $\mathrm{Li}$-ion conductivities.

\section{Critical Current Density}

Although significant advances have been made to improve the Li-ion conductivities of CEs, the improvement in the rate capability, which is needed for a practical all-solid-state battery, has been a challenge. Herein, we observed good cycling behavior for PEO:nwLLZO-10 and PEO:pLLZO-10, as indicated by a relatively constant polarization during each plating and stripping step. Figure 5D depicts the Nyquist plots of the symmetric cells before and after cycling and the corresponding equivalent circuit used to fit the spectra is shown as an inset. The Nyquist plots consist of a depressed semicircle associated with the Li/LLZO interface, which can be fitted by a $\mathrm{R}_{\text {int }} / \mathrm{CPE}$ element. The offset from the origin can be fitted by an additional $\mathrm{R}$ element which can be assigned to the resistance of the CE. The deduced area specific resistance (ASR) values, given by $\mathrm{ASR}=\mathrm{R}_{\text {int }} / 2 A$, are presented in Table 2. The critical current densities for PEO: pLLZO-10 and PEO:nwLLZO-10 were found in a range of $0.5 \mathrm{~mA} \mathrm{~cm}^{-2}$ to $1 \mathrm{~mA} \mathrm{~cm}^{-2}$, which are significantly higher than reported values for $\mathrm{CE}$ obtained at the same temperature or even higher temperatures (see Table 2). The explanation of the high CCD values obtained herein is not straightforward. There is no obvious correlation between particle content and distribution, particle morphology, temperature, Li-ion conductivity of the membrane, and the CCD by comparing the values reported in the literature and the findings as observed herein (see Table 2).

A systematic study varying all these parameters is needed to make any conclusions about the role of the individual factors on the rate capability of CEs in symmetrical Li cells. Overall, we suggest, for example, to use CEs containing fibrous garnet scaffolds that could allow the creation of a solid-state electrolyte framework with continuous Li-ion conducting pathways and a high surface volume ratio in CEs (Gong et al., s2018), wherein application of surface modifiers and suitable plasticizers could promote the fast $\mathrm{Li}$-ion transport and enable the high plating rates required for designing a practical all-solid-state Li metal battery for vehicle electrification.

\section{CONCLUSION}

This study examined the influence of LLZO filler morphology and concentration in PEO on the Li-ion conductivity and rate 
TABLE 2 | Critical current densities in comparison with values reported in the literature.

\begin{tabular}{|c|c|c|c|c|c|c|}
\hline LLZO (wt\%) & $\begin{array}{c}\text { Particle } \\
\text { distribution }\end{array}$ & $\mathbf{T}\left({ }^{\circ} \mathrm{C}\right)$ & $\sigma\left(\times 10^{-4} \mathrm{~S} \mathrm{~cm}^{-1}\right)$ & ASR $\left(\Omega \mathrm{cm}^{2}\right)$ & $C D\left(\mathrm{~mA} \mathrm{~cm} \mathrm{~cm}^{-2}\right)$ & Ref. \\
\hline 10 (particles) & Homogeneous & 70 & 4.47 & 33 & 0.1 & Zagórski et al. (2019) \\
\hline 5 (particles) & - & 30 & 7.70 & - & 0.05 & Karthik and Murugan, (2019) \\
\hline 70 (particles) & Percolating & 20 & 0.06 & 200 & $0.005-0.1$ & Keller et al. (2017) \\
\hline 10 (particles) & Homogeneous & 30 & 0.42 & - & $0.05-0.2$ & Li et al. (2020b) \\
\hline 10 (particles) & - & 60 & 2.0 & 233 & $0.3-0.5$ & Nguyen et al. (2020) \\
\hline 5 (nanowires) & No percolating network & 20 & 1.13 & $5 \times 10^{3}$ & 0.05 & Yang et al. (2017) \\
\hline 10 (particles) & - & $\mathrm{RT}$ & 0.03 & 325 & - & Cheng et al. (2017) \\
\hline 10 (particles) & Homogeneous & 60 & 2.04 & 266 & 0.5 & This work \\
\hline 10 (nanowires) & Homogeneous & 60 & 1.38 & 129 & 1.0 & This work \\
\hline
\end{tabular}

performance in symmetrical Li cells and synthesized two types of morphologies: particles and nanowires via ceramic sintering and electrospinning, respectively. PEO-based CE membranes with 10 and $20 \mathrm{wt} \%$ filler content were prepared by the solution cast process. The homogeneous distribution of the filler was confirmed without any indications of filler agglomerations by SEM on an ion-sliced cross section of the membrane and by $\mu$-XCT. The crystallinity of the membranes was investigated by XRD and DSC, indicating a decreased crystallinity for membranes with lower filler content due to the disruption of crystallinity in the PEO network. With higher filler concentration, the crystallinity increases again, which could be related to the excessive LLZO in the CEs that could hinder the segmental motion of the polymeric chains in the CEs. The alteration in crystallinity is also evident from impedance spectroscopy studies. A lower filler concentration, and hence lower crystallinity, is found to be associated with an improved ionic transport in the CE. Interestingly, no significant difference between particles and nanowires was observed. Overall, we did not find any evidence that the LLZO fillers take part in the overall Li-ion transport within the membrane, which might be related to the high interface resistance between the polymer/ceramic regions. Even when the filler takes an active part, it is questionable if this would lead to any improvement in the Liion conductivity. For example, when Li-ion transport would take place in well interconnected (but loosely bonded) LLZO particles, no improvement in terms of the overall Li-ion conductivity can be expected, since the conductivity of compressed LLZO powders (which can be considered as the maximum achievable value) is in orders of magnitude lower compared to sintered pellets (Zagórski et al., 2019), leading to similar conductivities than what is achievable for PEO without adding any filler. Further, the poor critical current densities $<0.5 \mathrm{~mA} \mathrm{~cm}^{-2}$ for CEs reported thus far indicate the electrochemical and mechanical instabilities of these electrolytes. We demonstrate that the optimized and uniformly distributed filler contents in CEs could improve these characteristics to achieve the critical current density of $1 \mathrm{~mA} \mathrm{~cm}^{-2}$. Overall, these results suggest that further research is needed to 1 ) reduce the crystallinity of PEO membranes by using modifiers or plasticizers and to 2) develop strategies to lower the resistance across the PEO/LLZO interface below a value of at least $100 \Omega \mathrm{cm}^{2}$ to enable the Li-ion transport through the polymer matrix and the percolating network formed by the ceramic fillers, which in turn could enable high-performing SSEs.

\section{DATA AVAILABILITY STATEMENT}

The raw data supporting the conclusions of this article will be made available by the authors, without undue reservation.

\section{AUTHOR CONTRIBUTIONS}

DR conceived and supervised the research work. MD performed the experimental work and data analysis and wrote the manuscript along with DR. $\mathrm{MH}$ assisted in electrospinning and manuscript preparation. SF, KR, and CS contributed to the preparation of polymer membranes and manuscript writing. FC and RB assisted in the analysis of micro-X-ray computed tomography data. $\mathrm{IH}$ and $\mathrm{VH}$ contributed to the manuscript preparation. All authors reviewed the manuscript.

\section{FUNDING}

Financial support by the Austrian Federal Ministry for Digital and Economic Affairs, the National Foundation for Research, Technology and Development, and the Christian Doppler Research Association (International Christian Doppler Laboratory for Solid-State Batteries and Christian Doppler Laboratory for Organocatalysis in Polymerization) is gratefully acknowledged. We acknowledge the financial support from the European Union (EU) under the Horizon 2020 research and innovation program (grant agreement No. 875514 “ECO²LIB”).

\section{ACKNOWLEDGMENTS}

We acknowledge support from B. Sartory and J. Wosik, both MCL, for the FESEM-EDX measurements, and J. Rosc for the $\mathrm{X}$-ray computed tomography measurements under the scope of the COMET program within the K2 Center "Integrated Computational Material, Process, and Product Engineering (IC-MPPE)" project ASSESS P1.10. 


\section{REFERENCES}

Balo, L., Shalu, H., Gupta, H., Kumar Singh, V., and Kumar Singh, R. (2017). Flexible Gel Polymer Electrolyte Based on Ionic Liquid EMIMTFSI for Rechargeable Battery Application. Electrochim. Acta 230, 123-131. doi:10.1016/j.electacta.2017.01.177

Baril, D., Michot, C., and Armand, M. (1997). Electrochemistry of Liquids vs. Solids: Polymer Electrolytes. Solid State Ion. 94 (1-4), 35-47. doi:10.1016/s01672738(96)00614-5

Bonizzoni, S., Ferrara, C., Berbenni, V., Anselmi-Tamburini, U., Mustarelli, P., and Tealdi, C. (2019). NASICON-type Polymer-In-Ceramic Composite Electrolytes for Lithium Batteries. Phys. Chem. Chem. Phys. 21 (11), 6142-6149. doi:10.1039/c9cp00405j

Brogioli, D., Langer, F., Kun, R., and La Mantia, F. (2019). Space-Charge Effects at the Li7La3Zr2O12/Poly(ethylene Oxide) Interface. ACS Appl. Mater. Inter. 11 (12), 11999-12007. doi:10.1021/acsami.8b19237

Chen, F., Yang, D., Zha, W., Zhu, B., Zhang, Y., Li, J., et al. (2017). Solid Polymer Electrolytes Incorporating Cubic Li7La3Zr2O12 for All-Solid-State Lithium Rechargeable Batteries. Electrochim. Acta 258, 1106-1114. doi:10.1016/ j.electacta.2017.11.164

Chen, L., Li, Y., Li, S.-P., Fan, L.-Z., Nan, C.-W., and Goodenough, J. B. (2018). $\mathrm{PEO} /$ garnet Composite Electrolytes for Solid-State Lithium Batteries: From "Ceramic-In-Polymer" to "Polymer-In-Ceramic". Nano Energy 46, 176-184. doi:10.1016/j.nanoen.2017.12.037

Cheng, S. H.-S., He, K.-Q., Liu, Y., Zha, J.-W., Kamruzzaman, M., Ma, R. L.-W., et al. (2017). Electrochemical Performance of All-Solid-State Lithium Batteries Using Inorganic Lithium Garnets Particulate Reinforced PEO/ LiClO4 Electrolyte. Electrochim. Acta 253, 430-438. doi:10.1016/ j.electacta.2017.08.162

Choi, J.-H., Lee, C.-H., Yu, J.-H., Doh, C.-H., and Lee, S.-M. (2015). Enhancement of Ionic Conductivity of Composite Membranes for All-Solid-State Lithium Rechargeable Batteries Incorporating Tetragonal Li7La3Zr2O12 into a Polyethylene Oxide Matrix. J. Power Sourc. 274, 458-463. doi:10.1016/ j.jpowsour.2014.10.078

Fu, K., Gong, Y., Dai, J., Gong, A., Han, X., Yao, Y., et al. (2016). Flexible, SolidState, Ion-Conducting Membrane with 3D Garnet Nanofiber Networks for Lithium Batteries. Proc. Natl. Acad. Sci. USA 113 (26), 7094-7099. doi:10.1073/ pnas. 1600422113

Gong, Y., Fu, K., Xu, S., Dai, J., Hamann, T. R., Zhang, L., et al. (2018). Lithium-ion Conductive Ceramic Textile: a New Architecture for Flexible Solid-State Lithium Metal Batteries. Mater. Today 21 (6), 594-601. doi:10.1016/ j.mattod.2018.01.001

Gupta, A., and Sakamoto, J. (2019). Controlling Ionic Transport through the PEOLiTFSI/LLZTO Interface. Electrochem. Soc. Interf. 28 (2), 63-69. doi:10.1149/ 2.f06192if

Kamaya, N., Homma, K., Yamakawa, Y., Hirayama, M., Kanno, R., Yonemura, M., et al. (2011). A Lithium Superionic Conductor. Nat. Mater 10 (9), 682-686. doi:10.1038/nmat3066

Karthik, K., and Murugan, R. (2019). Flexible High Li+ Conductive Lithium Garnet-Based Dry Solid Polymer Electrolyte Membrane with Enhanced Electrochemical Performance for Lithium Metal Batteries. Ionics 25 (10), 4703-4711. doi:10.1007/s11581-019-03044-y

Keller, M., Appetecchi, G. B., Kim, G.-T., Sharova, V., Schneider, M., Schuhmacher, J., et al. (2017). Electrochemical Performance of a Solvent-free Hybrid CeramicPolymer Electrolyte Based on Li 7 La 3 Zr 2 O 12 in P(EO) 15 LiTFSI. J. Power Sourc. 353, 287-297. doi:10.1016/j.jpowsour.2017.04.014

Kuhnert, E., Ladenstein, L., Jodlbauer, A., Slugovc, C., Trimmel, G., Wilkening, H. M. R., et al. (2020). Lowering the Interfacial Resistance in Li6.4La3Zr1.4Ta0.6O12|Poly(Ethylene Oxide) Composite Electrolytes. Cel Rep. Phys. Sci. 1 (10), 100214. doi:10.1016/j.xcrp.2020.100214

Langer, F., Bardenhagen, I., Glenneberg, J., and Kun, R. (2016). Microstructure and Temperature Dependent Lithium Ion Transport of Ceramic-Polymer Composite Electrolyte for Solid-State Lithium Ion Batteries Based on Garnet-type Li7La3Zr2O12. Solid State Ion. 291, 8-13. doi:10.1016/ j.ssi.2016.04.014

Li, J., Zhu, K., Yao, Z., Qian, G., Zhang, J., Yan, K., et al. (2020). A Promising Composite Solid Electrolyte Incorporating LLZO into PEO/PVDF Matrix for
All-Solid-State Lithium-Ion Batteries. Ionics 26 (3), 1101-1108. doi:10.1007/ s11581-019-03320-x

Li, W., Erickson, E. M., and Manthiram, A. (2020). High-nickel Layered Oxide Cathodes for Lithium-Based Automotive Batteries. Nat. Energ. 5 (1), 26-34. doi:10.1038/s41560-019-0513-0

Lin, C. W., Hung, C. L., Venkateswarlu, M., and Hwang, B. J. (2005). Influence of $\mathrm{TiO}_{2}$ Nano-Particles on the Transport Properties of Composite Polymer Electrolyte for Lithium-Ion Batteries. J. Power Sourc. 146 (1-2), 397-401. doi:10.1016/j.jpowsour.2005.03.028

Lin, D., Liu, W., Liu, Y., Lee, H. R., Hsu, P.-C., Liu, K., et al. (2016). High Ionic Conductivity of Composite Solid Polymer Electrolyte via In Situ Synthesis of Monodispersed SiO2 Nanospheres in Poly(ethylene Oxide). Nano Lett. 16 (1), 459-465. doi:10.1021/acs.nanolett.5b04117

Liu, W., Lee, S. W., Lin, D., Shi, F., Wang, S., Sendek, A. D., et al. (2017). Enhancing Ionic Conductivity in Composite Polymer Electrolytes with Well-Aligned Ceramic Nanowires. Nat. Energ. 2 (5), 1-7. doi:10.1038/ nenergy.2017.35

Liu, W., Liu, N., Sun, J., Hsu, P.-C., Li, Y., Lee, H.-W., et al. (2015). Ionic Conductivity Enhancement of Polymer Electrolytes with Ceramic Nanowire Fillers. Nano Lett. 15 (4), 2740-2745. doi:10.1021/acs.nanolett.5b00600

Long, L., Wang, S., Xiao, M., and Meng, Y. (2016). Polymer Electrolytes for Lithium Polymer Batteries. J. Mater. Chem. A. 4 (26), 10038-10069. doi:10.1039/ c6ta02621d

Murugan, R., Thangadurai, V., and Weppner, W. (2007). Fast Lithium Ion Conduction in Garnet-Type Li7La3Zr2O12. Angew. Chem. Int. Ed. 46 (41), 7778-7781. doi:10.1002/anie.200701144

Ngai, K. S., Ramesh, S., Ramesh, K., and Juan, J. C. (2016). A Review of Polymer Electrolytes: Fundamental, Approaches and Applications. Ionics 22 (8), 1259-1279. doi:10.1007/s11581-016-1756-4

Nguyen, Q. H., Luu, V. T., Nguyen, H. L., Lee, Y. W., Cho, Y., Kim, S. Y., et al. (2020). Li7La3Zr2O12 Garnet Solid Polymer Electrolyte for Highly Stable AllSolid-State Batteries. Front. Chem. 8, 619832. doi:10.3389/fchem.2020.619832

Rettenwander, D., Redhammer, G., Preishuber-Pflügl, F., Cheng, L., Miara, L., Wagner, R., et al. (2016). Structural and Electrochemical Consequences of Al and Ga Cosubstitution in Li7La3Zr2O12 Solid Electrolytes. Chem. Mater. 28 (7), 2384-2392. doi:10.1021/acs.chemmater.6b00579

Schneider, G. J. (2017). Dynamics of Nanocomposites. Curr. Opin. Chem. Eng. 16 65-77. doi:10.1016/j.coche.2017.04.006

Tan, S.-J., Zeng, X.-X., Ma, Q., Wu, X.-W., and Guo, Y.-G. (2018). Recent Advancements in Polymer-Based Composite Electrolytes for Rechargeable Lithium Batteries. Electrochem. Energ. Rev. 1 (2), 113-138. doi:10.1007/ s41918-018-0011-2

Vorauer, T., Kumar, P., Berhaut, C. L., Chamasemani, F. F., Jouneau, P. H., Aradilla, D., et al. (2020). Multi-scale Quantification and Modeling of Aged Nanostructured Silicon-Based Composite Anodes. Commun. Chem. 3 (1), 1-11. doi:10.1038/s42004-020-00386-x

Wan, Z., Lei, D., Yang, W., Liu, C., Shi, K., Hao, X., et al. (2019). Low ResistanceIntegrated All-Solid-State Battery Achieved by Li7 La3 Zr2 O12 Nanowire Upgrading Polyethylene Oxide (PEO) Composite Electrolyte and PEO Cathode Binder. Adv. Funct. Mater. 29 (1), 1805301. doi:10.1002/adfm.201805301

Wolfenstine, J., Ratchford, J., Rangasamy, E., Sakamoto, J., and Allen, J. L. (2012). Synthesis and High Li-Ion Conductivity of Ga-stabilized Cubic $\mathrm{Li}_{7} \mathrm{La}_{3} \mathrm{Zr}_{2} \mathrm{O}_{12}$. Mater. Chem. Phys. 134 (2-3), 571-575. doi:10.1016/ j.matchemphys.2012.03.054

Wu, J.-F., Chen, E.-Y., Yu, Y., Liu, L., Wu, Y., Pang, W. K., et al. (2017). Gallium-Doped Li7La3Zr2O12 Garnet-Type Electrolytes with High Lithium-Ion Conductivity. ACS Appl. Mater. Inter. 9 (2), 1542-1552. doi:10.1021/acsami.6b13902

Wu, X.-L., Xin, S., Seo, H.-H., Kim, J., Guo, Y.-G., and Lee, J.-S. (2011). Enhanced $\mathrm{Li}+$ Conductivity in PEO-LiBOB Polymer Electrolytes by Using Succinonitrile as a Plasticizer. Solid State Ion. 186 (1), 1-6. doi:10.1016/j.ssi.2011.01.010

Xue, Z., He, D., and Xie, X. (2015). Poly(ethylene Oxide)-Based Electrolytes for Lithium-Ion Batteries. J. Mater. Chem. A. 3 (38), 19218-19253. doi:10.1039/c5ta03471j

Yang, T., Zheng, J., Cheng, Q., Hu, Y.-Y., and Chan, C. K. (2017). Composite Polymer Electrolytes with Li7La3Zr2O12 Garnet-Type Nanowires as Ceramic Fillers: Mechanism of Conductivity Enhancement and Role of Doping and Morphology. ACS Appl. Mater. Inter. 9 (26), 21773-21780. doi:10.1021/ acsami.7b03806 
Zagórski, J., López del Amo, J. M., Cordill, M. J., Aguesse, F., Buannic, L., and Llordés, A. (2019). Garnet-Polymer Composite Electrolytes: New Insights on Local Li-Ion Dynamics and Electrodeposition Stability with Li Metal Anodes. ACS Appl. Energ. Mater. 2 (3), 1734-1746. doi:10.1021/ acsaem. 8 b0 01850

Zaman, W., Hortance, N., Dixit, M. B., De Andrade, V., and Hatzell, K. B. (2019). Visualizing Percolation and Ion Transport in Hybrid Solid Electrolytes for LiMetal Batteries. J. Mater. Chem. A. 7 (41), 23914-23921. doi:10.1039/ c9ta05118j

Zheng, J., and Hu, Y.-Y. (2018). New Insights into the Compositional Dependence of Li-Ion Transport in Polymer-Ceramic Composite Electrolytes. ACS Appl. Mater. Inter. 10, 4113-4120. doi:10.1021/acsami.7b17301

Zheng, J., Tang, M., and Hu, Y. Y. (2016). Lithium Ion Pathway within Li 7 La $3 \mathrm{Zr}$ 2 O 12 -Polyethylene Oxide Composite Electrolytes. Angew. Chem. 128 (40), 12726-12730. doi:10.1002/ange.201607539

Conflict of Interest: IH and VH are employed by AVL List GmbH. FC and RB are employed by Materials Center Leoben, Forschung GmbH.
The remaining authors declare that the research was conducted in the absence of any commercial or financial relationships that could be construed as a potential conflict of interest.

Publisher's Note: All claims expressed in this article are solely those of the authors and do not necessarily represent those of their affiliated organizations, or those of the publisher, the editors, and the reviewers. Any product that may be evaluated in this article, or claim that may be made by its manufacturer, is not guaranteed or endorsed by the publisher.

Copyright (๑ 2021 Din, Häusler, Fischer, Ratzenböck, Chamasemani, Hanghofer, Henninge, Brunner, Slugove and Rettenwander. This is an open-access article distributed under the terms of the Creative Commons Attribution License (CC $B Y)$. The use, distribution or reproduction in other forums is permitted, provided the original author(s) and the copyright owner(s) are credited and that the original publication in this journal is cited, in accordance with accepted academic practice. No use, distribution or reproduction is permitted which does not comply with these terms. 